\title{
Graphene coatings: An efficient protection from oxidation
}

\author{
M. Topsakal, ${ }^{1,2}$ H. Şahin, ${ }^{1,2}$ and S. Ciraci ${ }^{1,2,3, *}$ \\ ${ }^{1}$ UNAM-National Nanotechnology Research Center, Bilkent University, 06800 Ankara, Turkey \\ ${ }^{2}$ Institute of Materials Science and Nanotechnology, Bilkent University, 06800 Ankara, Turkey \\ ${ }^{3}$ Department of Physics, Bilkent University, 06800 Ankara, Turkey
}

(Received 6 November 2011; revised manuscript received 12 February 2012; published 23 April 2012)

\begin{abstract}
We demonstrate that graphene coating can provide efficient protection from oxidation by posing a high-energy barrier to the path of oxygen atom, which could have penetrated from the top of the graphene to the reactive surface underneath. A graphene bilayer, which blocks the diffusion of oxygen with a relatively higher energy barrier, provides even better protection from oxidation. While an oxygen molecule is weakly bound to a bare graphene surface and hence becomes rather inactive, it can easily dissociate into two oxygen atoms adsorbed to low-coordinated carbon atoms at the edges of a vacancy. For these oxygen atoms the oxidation barrier is reduced and hence the protection from oxidation provided by graphene coatings is weakened. Our predictions obtained from the state-of-the-art first-principles calculations of the electronic structure, phonon density of states, and reaction path will unravel how graphene can be used as a corrosion-resistant coating and guide further studies aimed at developing more efficient nanocoatings.
\end{abstract}

DOI: 10.1103/PhysRevB.85.155445

PACS number(s): 81.16.Pr, 68.65.Pq, 66.30.Pa, 81.05.ue

\section{INTRODUCTION}

The reaction of material surfaces with oxygen and controlling damages from corrosion have been the subject of intensive research for decades. While protective macroscale coatings give rise to modification of the sizes and some other physical properties of reactive surfaces, progress made to date has revealed several advantages of nanoscale coatings in protection from corrosion and wear.

The earliest efforts on protection from oxidation using carbon-based materials were devoted to carbon deposition on metal surfaces. It was reported that $\mathrm{Ni}$ and $\mathrm{Co}$ surfaces can be covered by carbon deposition at high temperatures. ${ }^{1}$ It was shown that the (111) surfaces of $\mathrm{Ni}$ single crystals can be covered by monolayer carbon as a result of carbon segregation through the metal surface. ${ }^{2}$ Soon after, the surface segregation behavior of carbon from dilute solid solutions on $\operatorname{Pt}(100), \operatorname{Pt}(111), \operatorname{Pd}(100), \operatorname{Pd}(111)$, and Co(0001) surfaces was investigated. ${ }^{3}$ Interestingly, much earlier it was argued that the segregated carbon layer can be in the form of a monolayer honeycomb structure like graphene. ${ }^{4}$

Graphene, ${ }^{5}$ being not only the thinnest ever but also the strongest material, has, in fact, the potential for nanocoating applications. When stuck to or grown on various surfaces, graphene adds only a negligible thickness to the size of the underlying sample and forms an electrically and thermally conductive coating on it. Moreover, graphene has exceptional mechanical, thermal, and chemical stability. Various synthesis techniques of graphene covered metal surfaces and their electronic and structural properties have been reviewed by Winterlin et al. ${ }^{6}$ and Mattevi et al. ${ }^{7}$ Advances in the techniques of graphene synthesis have initiated studies on graphene coating. Experimentally, Dedkov et al. ${ }^{8}$ studied the oxygen protection of $\mathrm{Fe}$ intercalated $\mathrm{Ni}$ surfaces and bare $\mathrm{Ni}$ films. Borca et al. $^{9}$ have experimentally demonstrated that the periodically rippled structure of graphene can be grown on $\mathrm{Ru}(0001)$ surface and it serves as a perfect coating material against oxidation. Very recently, Gadipelli et al. ${ }^{10}$ reported the formation of large-scale graphene monolayers on a $\mathrm{Cu}$ surface, which is well protected from oxidation. Also, graphene coatings on $\mathrm{Cu}, \mathrm{Cu} / \mathrm{Ni}$ alloy, $\mathrm{Pt}$, and $\mathrm{Ir}$ surfaces have been exploited. ${ }^{11-13}$ XPS and SEM images presented evidence that $\mathrm{Cu}$ and $\mathrm{Cu} / \mathrm{Ni}$ surfaces can be protected from oxidation through graphene coating. ${ }^{11}$ However, despite this recent progress, very little is known about how and why a graphene layer constitutes a protective coating on reactive surfaces and what its limitations are.

In this study we show that graphene can easily be oxidized by oxygen atoms, which form strong chemical bonds on its surface. Despite this, the graphene coating can protect solid surfaces from oxidation by posing a high-energy barrier to any adsorbed oxygen atom diffusing from the top of the graphene to the interface between the graphene and the reactive surface underneath. Because of this barrier, the perpendicular diffusivity of oxygen atoms is practically 0 compared to its lateral diffusivity. Although an oxygen molecule is weakly bound to graphene and does not have any direct role in oxidation, it can be indirectly involved by dissociating into two atomic oxygens. These oxygen atoms form relatively stronger chemical bonds with twofold coordinated carbons but encounter a much lower oxidation barrier when they diffuse toward the reactive surface. Poor protection from oxidation at defect sites can be circumvented by a multilayer graphene coating.

\section{METHOD}

Our study proceeds in three complementary and sequential steps. (i) In the first step we examine the interaction of $\mathrm{O}_{2}$ and $\mathrm{O}$ atoms with a bare reactive metal surface and with a bare pristine graphene, where important features are discovered. (ii) The second step deals with the sticking of graphene to a flat, clean surface, which is vulnerable to oxidation. (iii) In the third step, we show how a graphene coating hinders oxygen atoms from diffusion toward the protected surface. Our results are obtained by performing first-principles, spin-polarized calculations within density functional theory using the VASP package. ${ }^{14,15}$ We used the generalized gradient approximation ${ }^{16}$ including Van der Waals (vdW) correction, ${ }^{17}$ PAW potentials, ${ }^{18}$ and a 
plane-wave basis set with a kinetic energy cutoff of $500 \mathrm{eV}$. To minimize the coupling between adsorbed atoms or molecules, the binding energies and reaction paths are calculated using $(4 \times 4)$ or $(6 \times 6)$ supercells. For coated surfaces a grid of $25 \times 25 \times 1 \mathbf{k}$ points is used. The convergence criterion of selfconsistent calculations for ionic relaxations is taken to be $10^{-5}$ $\mathrm{eV}$ between two consecutive steps. By using the conjugate gradient method, atomic positions and lattice constants are optimized until the atomic forces are less than $0.05 \mathrm{eV} / \AA$. The energetics of various paths of $\mathrm{O}$ or $\mathrm{O}_{2}$ are calculated by forcing them to pass through the graphene layer from above to below. The amount of displacement is identified as indentation in the figures. The paths of minimum energy barrier are determined by relaxing carbon atoms of graphene, as well as lateral $x$ and $y$ coordinates of $\mathrm{O}$ or $\mathrm{O}_{2}$ at each step of indentation corresponding to a fixed $z$ coordinate. In some cases, the energy barriers associated with specific and well-determined paths are also examined, where $\mathrm{O}_{2}$ and $\mathrm{O}$ are forced to follow these paths, but the rest of atoms are relaxed. The maximum number of atoms treated in our calculations is 129, which occurred in the determination of energy barriers associated with the coating of a $\mathrm{Al}(111)$ surface by graphene bilayer.

\section{OXIDATION OF ALUMINUM SURFACE AND GRAPHENE}

Since we are not concerned with sample specific details of oxidation behaviors of the protected surfaces, the $\mathrm{Al}(111)$ surface is taken here only as a prototype metal surface vulnerable to oxidation when exposed to the atmosphere but is protected by placing a graphene sheet between its surface and atmosphere. The $\mathrm{Al}(111)$ surface alone is represented by a four-layer $\mathrm{Al}(111) \mathrm{slab}$ as shown in Fig. 1. It has metallic and nonmagnetic ground states, and its states at the Fermi level $\left(E_{F}\right)$ are composed of mainly $3 p_{x y}$ and partially $3 s$ orbitals of $\mathrm{Al}$ atoms. The work function for this slab is calculated to be $4.06 \mathrm{eV}$, which is comparable to the value of $4.24 \mathrm{eV}$ measured experimentally ${ }^{19}$ for an $\mathrm{Al}(111)$ surface. We calculate that an oxygen atom is strongly bound to the
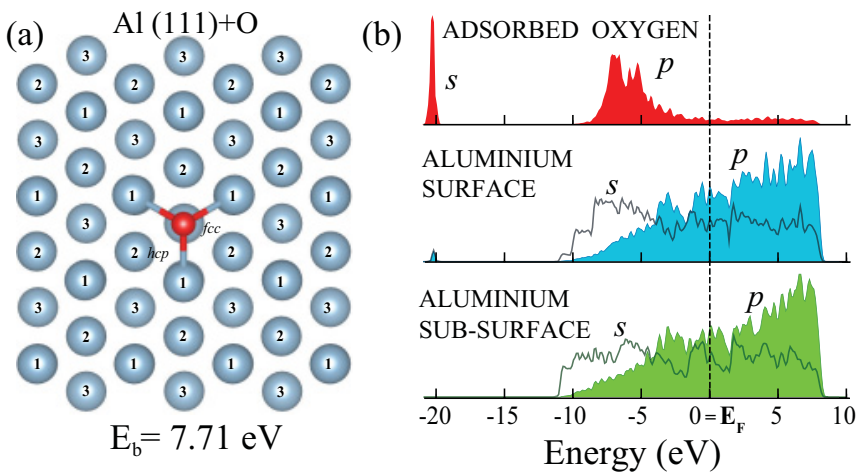

FIG. 1. (Color online) (a) Atomic configuration of an oxygen atom adsorbed to an $\mathrm{Al}(111)$ surface. Oxygen and $\mathrm{Al}$ atoms are illustrated by small (red) and large (blue) balls, with numerals indicating their layer numbers from the top. (b) Density of states projected to $s$ and $p$ orbitals of adsorbed $\mathrm{O}$ and surface and subsurface layers of an $\mathrm{Al}(111)$ slab.
$\mathrm{Al}(111)$ surface, with a 7.71-eV binding energy at the fcc site and $7.24 \mathrm{eV}$ at the hcp site. Figure 1(b) presents the densities of electronic states projected to adsorbate $\mathrm{O}$ and surface and subsurface layers of the $\mathrm{Al}(111)$ slab. Apparently, $2 p$ orbitals of adsorbed $\mathrm{O}$ mix with the $3 p$ and $3 s$ orbitals of the $\mathrm{Al}$ substrate over a wide energy range to form a strong bond. The oxygen molecule by itself interacts strongly with the $\mathrm{Al}(111)$ surface; it dissociates into atomic oxygens, which, in turn, are adsorbed at fcc and hep sites.

Bare graphene can also be easily oxidized, whereby oxygen atoms are adsorbed at the bridge-site positions above any $\mathrm{C}-\mathrm{C}$ bond of graphene and become negatively charged. Our calculations using Bader analysis ${ }^{20}$ estimate an excess charge of 0.79 electron at the adsorbed $\mathrm{O}$ atom. The binding energy is calculated to be $2.43 \mathrm{eV}$ at the bridge site, hence $\mathrm{O}$ atoms at the $\mathrm{T}$ (top) or $\mathrm{H}$ (hollow; i.e., center of hexagon) site in Fig. 2(a) move favorably to the B (bridge) site. Figure 2(c) presents the electronic energy band structure corresponding to an $\mathrm{O}$ atom adsorbed to a bridge site of a $(4 \times 4)$ supercell of graphene and the charge density distributions of specific conduction and valence band states. Upon oxidation the linearly crossing $\pi$ and $\pi^{*}$ states of semimetallic bare graphene are modified. While they cross at a specific $\vec{k}$-point along one type of $\mathrm{M}-\mathrm{K}$ direction at the Fermi level, they open a band gap of $0.58 \mathrm{eV}$ along $\mathrm{K}-\Gamma$ direction of Brillouin zone. However, Oxygen coverage, which breaks the hexagonal symmetry renders semimetallic graphene insulator. This explains why domains of dark (metallic) graphene surface become reflecting (insulator) upon oxidation. ${ }^{21}$ Reversible oxidation-deoxidation of graphene through heating or charging has been pointed out as a potential electronic device application. ${ }^{21,22}$ Reversibility is strong evidence that the graphene surface remains chemically stable in the course of oxidation-deoxidation; neither bond breaking nor modification of the honeycomb structure occurs. However, the situation is dramatically different for several other surfaces, such as $\mathrm{Si}, \mathrm{Fe}, \mathrm{Te}, \mathrm{Al}$, and $\mathrm{Cu}$, where the chemical stability is destroyed upon oxidation. ${ }^{11,23-25}$ Figure 2(d) shows the density of phonon modes of a pristine graphene and that of oxygen adsorbed to the bridge site of a $(4 \times 4)$ supercell of graphene, which are calculated from the first principles. ${ }^{26}$ As shown, the adsorbed oxygen atom gives rise to several localized phonon modes, which will be used in estimating the characterized frequency and the diffusivity thereof.

In contrast to an oxygen atom, an oxygen molecule shows weak binding with graphene. We calculated its binding energy to be $115 \mathrm{meV}$ and its magnetic moment $1.90 \mu_{B}$, slightly smaller than the magnetic moment of free $\mathrm{O}_{2}$. Hence, an $\mathrm{O}_{2}$ molecule with such a weak binding energy to bare graphene cannot have any significant effect on the oxidation of the protected surface, though the situation can be different for defective graphene, as we show later. On the other hand, a free $\mathrm{O}$ atom approaching another $\mathrm{O}$ atom already adsorbed on graphene forms a strong bond with the latter and releases $\sim 4.13 \mathrm{eV}$ energy in this exothermic process. Eventually an $\mathrm{O}_{2}$ molecule is formed thereof. This may explain why deoxidation of graphene is provided easily ${ }^{21}$ by an STM tip at $100^{\circ} \mathrm{C}$ in close proximity to an oxidized graphene surface, despite the strong binding energy of the $\mathrm{O}$ atom. Note that two adsorbed $\mathrm{O}$ atoms in close proximity can also form an $\mathrm{O}_{2}$ molecule by 


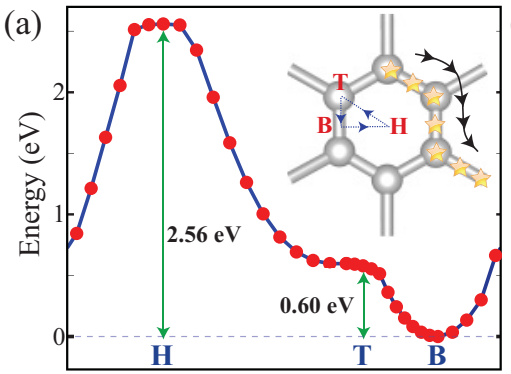

(b)

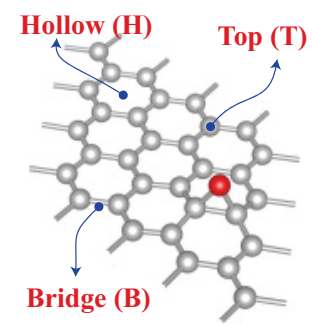

(c)
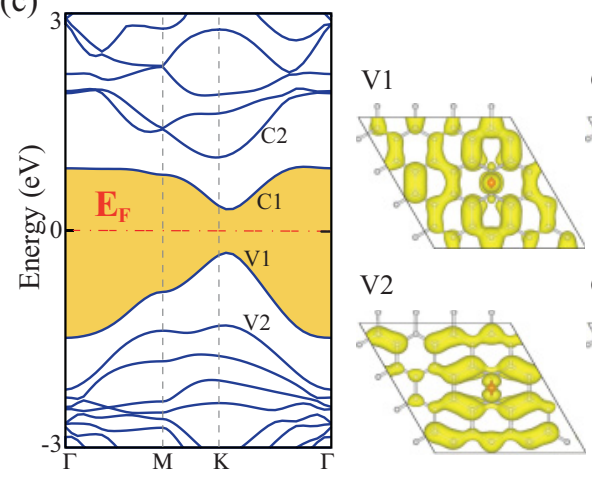

$\mathrm{C} 1$
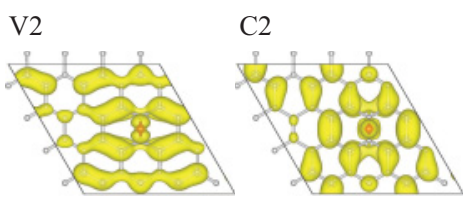

(d)

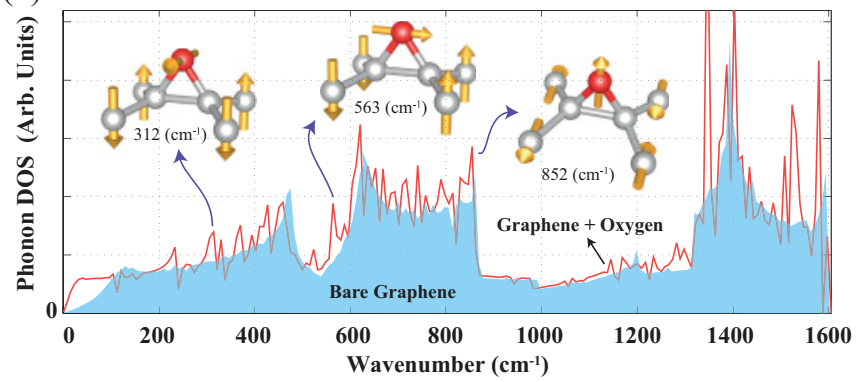

FIG. 2. (Color online) (a) Variation of the energy of adsorbed oxygen atom along the $\mathrm{T}$ (top) $-\mathrm{H}$ (hollow)-B( bridge) site directions of a hexagon showing that the $\mathrm{B}$ site is energetically most favorable. Stars indicate a favorable path for the diffusion of oxygen atom on the graphene surface. (b) Atomic configuration for an oxygen atom adsorbed at the bridge site on a $(4 \times 4)$ supercell of graphene consisting of 32 carbon atoms. (c) Electronic energy band structure together with charge densities of specific conduction and valence bands states. The crossing of $\pi$ and $\pi^{*}$ bands is clarified in the text. (d) Calculated density of phonon modes of a pristine graphene (shaded area) and those of oxygen adsorbed to the bridge site of the $(4 \times 4)$ supercell of graphene [solid (red) line]. Insets: Relevant localized phonon modes.

releasing an energy of $1.60 \mathrm{eV}$, if they can overcome the energy barrier.

\section{PROTECTION OF AN AL(111) SURFACE BY GRAPHENE COATING}

Next we explore the protection of a reactive surface, such as $\mathrm{Al}(111)$, by sticking graphene to it or growing graphene on it. Sticking of graphene to various metal surfaces including $\mathrm{Al}(111)$ surfaces has been studied earlier. ${ }^{27}$ Even though an $\mathrm{Al}(111)$ surface is not in registry with the graphene honeycomb structure and has hexagonal lattice constants $\sim 10 \%$ larger than those of graphene, sticking of graphene to this surface can be achieved. In order to present an estimation value for the adsorption energy of graphene to an $\mathrm{Al}(111)$ surface, one has to compress an $\mathrm{Al}(111)$ slab laterally and expand the graphene lattice in order to achieve a registry for the optimization of final structures using the periodic boundary conditions. Despite the strain energy spent to obtain the lattice registry, sticking occurs with the significant binding energy of $2.67 \mathrm{eV}$ per $(4 \times 4)$ supercell (or $\sim 166 \mathrm{meV}$ per cell). The sticking of graphene patches to an $\mathrm{Al}(111)$ surface in a random orientation is a complex and stochastic process and can even lead to the formation of bubbles, since low coordinated edge atoms show stronger binding to the $\mathrm{Al}(111)$ surface. Even if the average binding energy per carbon atom is low, it would require significant energy to peal off the strong but flexible graphene layer from the surface.

If graphene patches are placed randomly on $\mathrm{Al}(111)$, they may not be severely strained to maintain the lattice registry. Therefore, we instead consider unstrained graphene and compensate the lattice misfit by laterally compressing the $\mathrm{Al}(111)$ slab. In this way we achieve the lattice registry to be able to use the periodic boundary conditions. In these circumstances, the adsorption energy of graphene to this compressed $\mathrm{Al}(111)$ slab, which is calculated to be $2.38 \mathrm{eV}$ per $(4 \times 4)$ supercell (or $\sim 148 \mathrm{meV}$ per cell), is not significantly affected. Additionally, the binding energy of an $\mathrm{O}$ atom to the compressed $\mathrm{Al}(111)$ surface $(7.15 \mathrm{eV})$ is still very high. Thus, despite the compression dictated by the periodic boundary conditions, the compressed $\mathrm{Al}(111)$ slab is still sufficiently reactive to mimic a surface to be protected by graphene coating.

\section{A. Diffusion of $\mathrm{O}_{2}$ and $\mathrm{O}$ through suspended graphene}

Now we address the main issue, pertaining to how a graphene coating, which by itself is also vulnerable to oxidation, can protect a reactive metal surface. To clarify the mechanism of protection from oxidation, we first examine how an $\mathrm{O}_{2}$ molecule or $\mathrm{O}$ atom can pass from one side of bare and suspended graphene to the other side. The energetics and energy barriers involved in the course of these processes are calculated in a $(4 \times 4)$ supercell of bare graphene with specific carbon atoms fixed to prevent the suspended layer from displacement. Here we consider first the fixed vertical path passing through the hole at the center of hexagons (as a seemingly possible diffusion path) and calculate the involved energy barrier when an $\mathrm{O}_{2}$ molecule or $\mathrm{O}$ atom is forced to follow this path as summarized in Figs. 3(a) and 3(b). An oxygen molecule following this vertical path needs to overcome a barrier of $10.12 \mathrm{eV}$ in Fig. 3(a). However, once the $\mathrm{O}_{2}$ overcomes this barrier, it dissociates into two $\mathrm{O}$ atoms: one $\mathrm{O}$ is adsorbed above, and the other adsorbed below, at the bridge sites. Apparently, graphene acts as a membrane that blocks the passage of $\mathrm{O}_{2}$. If an $\mathrm{O}$ atom were forced to pass from the top to the bottom side along this fixed vertical path, the energy barrier would be even higher, i.e., $16.34 \mathrm{eV}$ in Fig. 3(b). This fixed vertical path is not a possible diffusion path with a minimum energy barrier, since there are reaction paths with lower energy barriers as explained below.

The path shown in Fig. 3(c) starts from the adsorbed oxygen atom at the bridge site on the top side, i.e., the minimum energy 


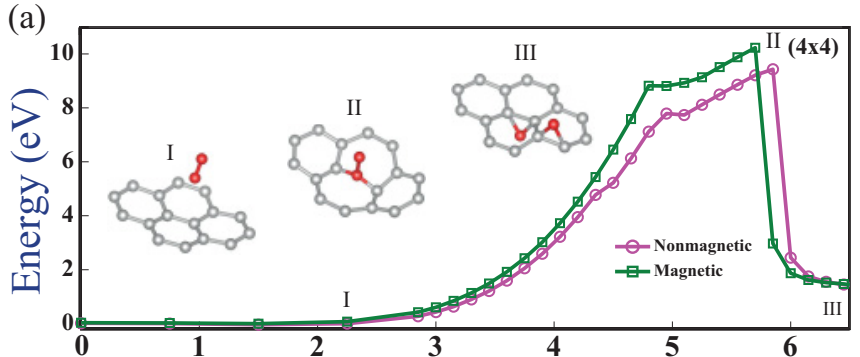

(b)
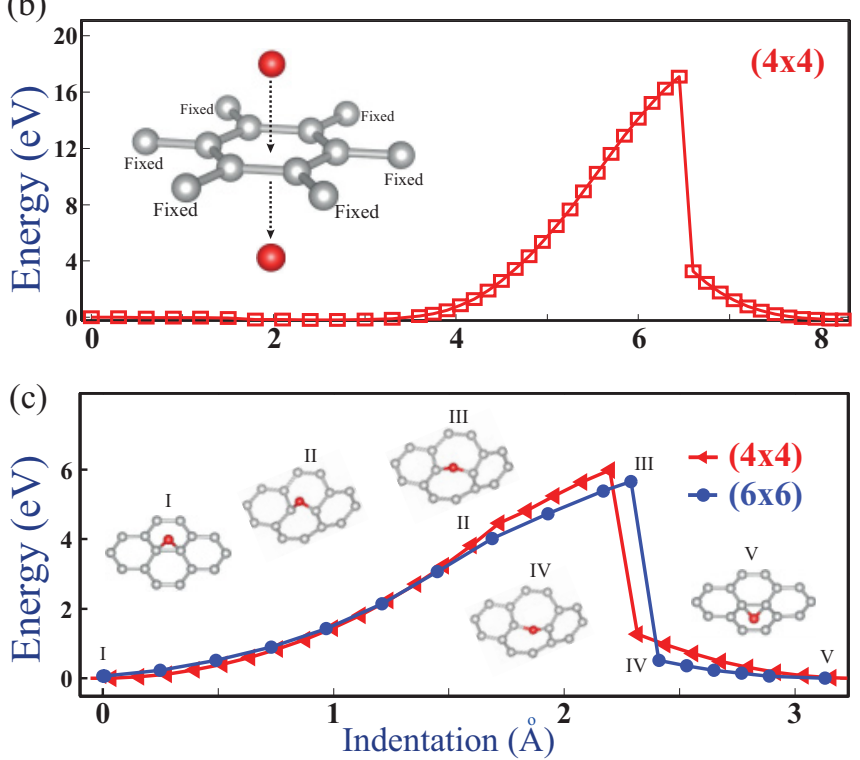

FIG. 3. (Color online) Calculation of energy barriers of $\mathrm{O}_{2}$ and $\mathrm{O}$ passing from the top to the bottom side of suspended graphene along various paths. (a) Energy barriers for triplet (magnetic) and singlet (nonmagnetic) $\mathrm{O}_{2}$, which are forced to pass from the top to the bottom side of graphene. (b) Energy barrier for an $\mathrm{O}$ atom along the same path as in (a). (c) The path of the minimum energy barrier for an $\mathrm{O}$ atom, which is initially adsorbed at the bridge site above the graphene plane, is forced to pass to the bottom side. Positions of $\mathrm{C}$ atoms, as well as the lateral $x$ and $y$ coordinates of $\mathrm{O}$, are optimized for each value of indentation.

configuration of an $\mathrm{O}$ atom adsorbed on pristine graphene. At each stage of indentation, carbon atoms, as well as $x$ and $y$ lateral coordinates of the $\mathrm{O}$ atom, are optimized to minimize the energy. As shown by the snapshots of atomic configurations corresponding to various stages, the passage of $\mathrm{O}$ takes place around the same bridge bond, whereby the $\mathrm{O}$ atom switches from the top to the bottom side of graphene by gradually flattening the $\mathrm{C}-\mathrm{O}-\mathrm{C}$ bridge bond. The energy barrier to be overcome by an $\mathrm{O}$ atom in order to pass from the top to the bottom side is calculated to be $Q=5.98 \mathrm{eV}$. To reveal whether the $(4 \times 4)$ supercell may impose constraints on the calculated energy barrier, we calculated the barrier in a relatively larger, $(6 \times 6)$ graphene supercell to be $Q=5.65 \mathrm{eV}$. The calculated energy barrier is not affected by the size of the supercell and is high enough to block diffusion and hence to hinder oxidation of the surface underneath. This path is identified as the path of the minimum energy barrier for an $\mathrm{O}$ atom passing from the top to the bottom of bare suspended graphene.
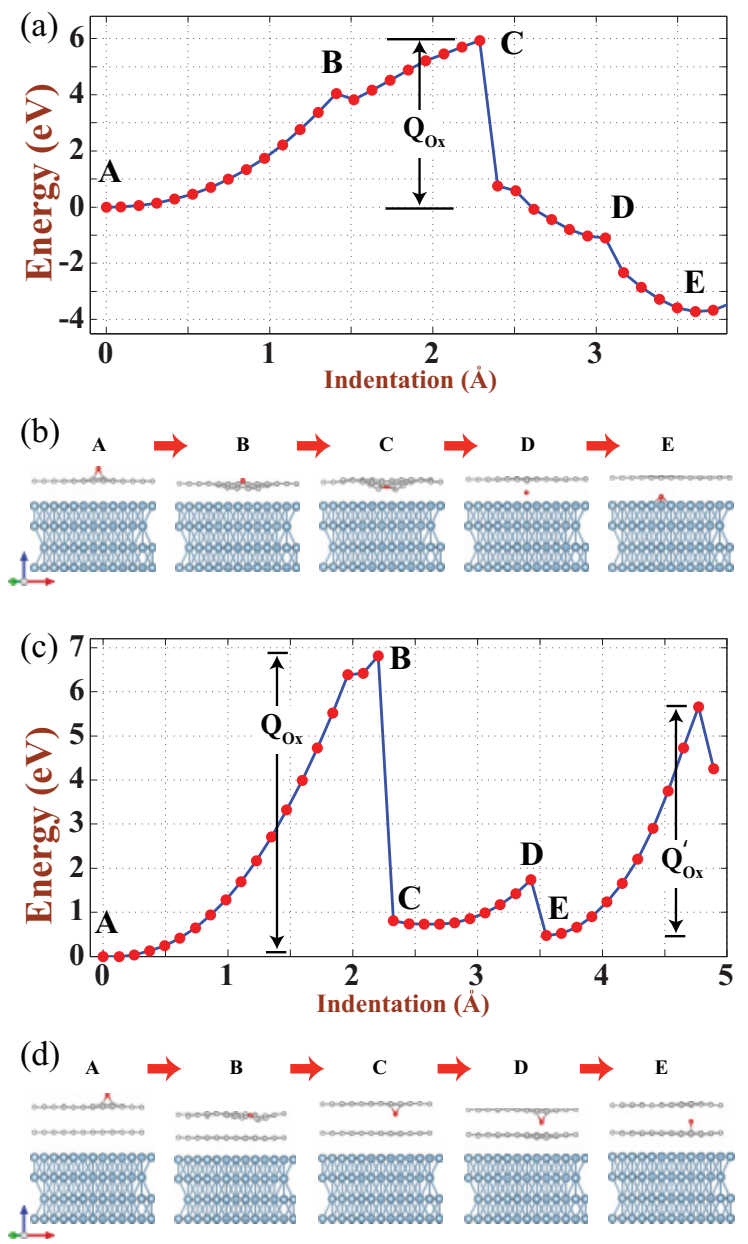

FIG. 4. (Color online) (a) Variation of the total energy for an $\mathrm{O}$ atom (red ball) passing (indenting) from the top side of a single graphene layer to its bottom side and eventually adsorbing to the $\mathrm{Al}(111)$ surface (blue balls) underneath. The $\mathrm{O}$ atom follows the path of the minimum energy barrier $\left(Q_{\mathrm{ox}}=5.93 \mathrm{eV}\right)$. (b) Snapshots of atomic configurations corresponding to various stages between the initial stage A, starting from the bridge site of graphene, and the final stage $\mathrm{E}$, ending with the adsorption of the $\mathrm{O}$ atom on $\mathrm{Al}(111)$. (c) Protection of an $\mathrm{Al}(111)$ surface from oxidation by a graphene bilayer and variation of energy for an $\mathrm{O}$ atom penetrating from the top side of the outermost graphene layer and eventually adsorbing to $\mathrm{Al}(111)$. The greatest barrier to be overcome by a diffusing $\mathrm{O}$ atom is $Q_{\text {ox }}=6.81 \mathrm{eV}$ along the the path to reach the $\mathrm{Al}(111)$ surface. (d) Atomic configurations of various stages of case (c).

\section{B. Graphene coating of $\mathrm{Al}(111)$}

In the presence of an $\mathrm{Al}(111)$ slab underneath the protective graphene coating, we elaborate and further optimize the reaction path in Fig. 3(c) as the most likely pathway of oxidation. The variation of the energy of an $\mathrm{O}$ atom moving along the reaction path of the minimum barrier is shown in Fig. 4(a). The energy barrier along this reaction path is $Q_{\text {ox }} \sim 5.93 \mathrm{eV}$ and occurs as $\mathrm{O}$ is switching from the top to the bottom side of graphene. Once the diffusing $\mathrm{O}$ atom overcomes this barrier, it goes to the $\mathrm{Al}(111)$ surface via the bridge site below with almost no barrier and oxidizes the metal surface. This energy barrier $Q_{\mathrm{ox}}$ is rather high and hence the 
protection of graphene against oxidation appears to be very efficient. The significance of the high $Q_{\text {ox }}$ can be deduced by comparing the diffusivity of an $\mathrm{O}$ moving on the graphene surface, $D_{\|}$, with that of an $\mathrm{O}$ atom penetrating the graphene coating to oxidize the $\mathrm{Al}(111)$ surface, $D_{\perp} . D_{\|}=a^{2} v e^{Q / k_{B} T}$ can be estimated in terms of the energy barrier $Q=0.60 \mathrm{eV}$ in Fig. 2(a), the lattice constant $a=1.43 \AA$, and the characteristic vibration frequency extracted from the calculated localized phonon modes of $\mathrm{O}$ in Fig. 2(d) is $v \cong 22 \mathrm{THz}$. Accordingly, the diffusivity of an $\mathrm{O}$ atom penetrating the graphene coating is estimated to be $D_{\perp}=D_{\|} \times 10^{-87}$, which is really negligible.

\section{Bilayer graphene coating of $\mathrm{Al}(111)$}

The effectiveness of the protection against oxidation can be further increased by coating with a graphene bilayer. In Fig. 4(c) the variation in energy of a diffusing $\mathrm{O}$ atom from the outermost graphene bilayer to the metal surface via a second graphene layer following the minimum energy reaction path is shown. Apparently, the oxidation barrier is increased by $0.88 \mathrm{eV}$ due to to the coating by a graphene bilayer. Snapshots of relevant stages in the course of diffusion of $\mathrm{O}$, starting above the first graphene layer through the second graphene layer and eventually ending at the $\mathrm{Al}(111)$ surface, are also shown in Fig. 4(d). While the oxidation barrier $Q_{\mathrm{ox}}=6.81 \mathrm{eV}$ occurs when the diffusing $\mathrm{O}$ switches from the top to the bottom side of the first graphene layer, there are additional barriers blocking the diffusion of $\mathrm{O}$ atoms. For example, the energy barriers for switching from the bottom bridge site of the first graphene layer to the top bridge site of the second graphene layer is $\sim 1 \mathrm{eV}$. If this small barrier is overcome, the adsorbed $\mathrm{O}$ atom becomes attached to the second graphene layer and is still separated from reactive surface. To proceed with diffusion to reach the $\mathrm{Al}(111)$ surface, one follows a course similar to that in Fig. 4(a). Starting from stage E, an $\mathrm{O}$ atom diffuses from the bridge site above the second graphene layer to the $\mathrm{Al}(111)$ surface by overcoming an energy barrier of $Q_{\mathrm{ox}}^{\prime}=5.20 \mathrm{eV}$ and oxidizes the metal surface.

Clearly, the coating of reactive surfaces by sheets comprising more than two layers of graphene will further increase the effectiveness of protection. Sequential barriers posed at each graphene layer increase the chance that the diffusing $\mathrm{O}$ can be trapped between any two barriers. On the other hand, the size of the protected sample will be modified by each additional graphene layer, adding $\sim 3 \AA$ distance between the $\mathrm{O}$ and the $\mathrm{Al}$ surface, even if this increase in thickness may be considered negligible. Protection by graphene sheets comprising a few layers is expected to be effective also to suppress the effects of any local heating or energy transfer to the outermost graphene. For example, as pointed out at the beginning, a free oxygen in close proximity to an adsorbed oxygen on graphene can form $\mathrm{O}_{2}$, whereby an energy of $\sim 4.13 \mathrm{eV}$ is released. When deposited on the graphene, this energy may create a local, nonequilibrium phonon distribution, which has been shown to dissipate within picoseconds. ${ }^{28,29}$ Such a short time interval is enough to accommodate several jumps of atoms. Hence local heating due to a chemical process may promote the diffusion of other adsorbed oxygen atoms from the protective coating toward the reactive surface. In these circumstances while a single-layer graphene coating fails to hinder oxidation, a multilayer graphene coating can block diffusing hot oxygen atoms.

\section{Vacancy effect}

The above arguments related to protection against oxidation rely on the fact that the graphene coating is continuous and defect-free. If the coating consists of graphene patches, reactive surfaces cannot be covered at the zones between patches, where they become directly exposed to atomic oxygens. ${ }^{11}$ Holes or vacancies ${ }^{30,31}$ of graphene are also spots where oxygen atoms would penetrate the metal surface without
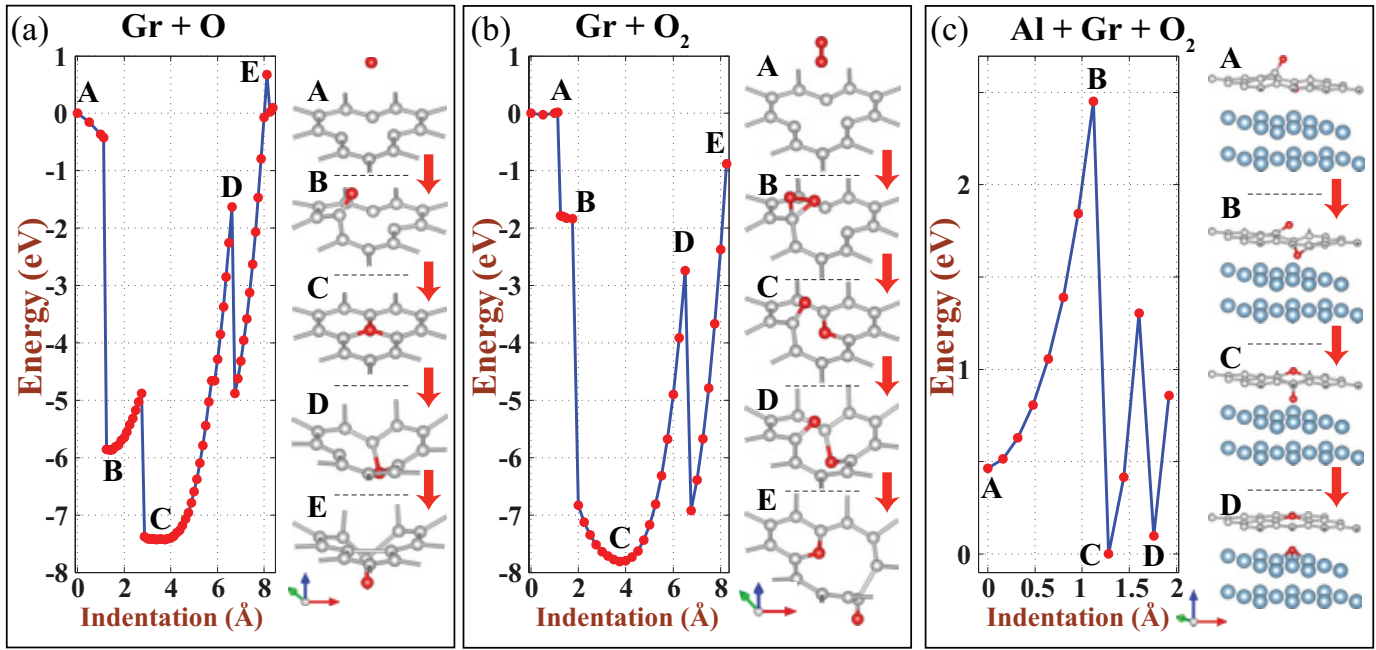

FIG. 5. (Color online) (a) Evolutions of energetics and atomic structure with the indentation of the oxygen atom, which is initially adsorbed at the edge of a single vacancy. (b) Evolutions of energetics and atomic structure of two oxygen atoms resulting from the dissociation of an $\mathrm{O}_{2}$ molecule at the edge of a single vacancy and the indentation of one of the oxygen atoms from the top to the bottom site of suspended graphene. (c) Diffusion of one of the adsorbed oxygen atoms in (b) toward the Al(111) surface, resulting in its oxidation. Red, gray, and blue balls indicate $\mathrm{O}, \mathrm{C}$, and $\mathrm{Al}$ atoms, respectively. 
or with a relatively smaller energy barrier. In fact, the etching of graphite following the dissociation of $\mathrm{O}_{2}$ by production of $\mathrm{CO}$ and $\mathrm{CO}_{2}$ has been pointed out. ${ }^{32,33}$ Here we consider the penetration of an oxygen atom near a vacancy in graphene. Three important features of our work are schematically summarized in Fig. 5. An oxygen atom can favorably bind to carbon atoms at the edge of a single vacancy in Fig. 5(a). The ground state is exothermic and releases $7.65 \mathrm{eV}$, whereby the $\mathrm{O}$ atom substitutes the vacant carbon atom. In Fig. 5(b) an $\mathrm{O}_{2}$ molecule can dissociate into two $\mathrm{O}$ atoms in close proximity to a vacancy in graphene. Subsequently, while one $O$ atom is attached to one of three twofold coordinated carbon atoms at the edge of the vacancy, the other one bridges between the remaining two and hence completes the hexagon. In this exothermic process $7.83 \mathrm{eV}$ energy is released, in addition to the energy spent in dissociation process. This shows that vacancies of graphene are active sites for catalysis of the dissociation of $\mathrm{O}_{2}$ molecules. Figure 5(c) shows that the barrier in the diffusion of a specific $\mathrm{O}$ atom adsorbed at the edge of a vacancy is dramatically lowered $\left(Q_{\mathrm{ox}} \sim 2 \mathrm{eV}\right)$ and hence the protection from oxidation is weakened. Such a situation occurs at the grain boundaries and holes of graphene and confirms the experiment $^{11}$ that defects or discontinuities in the covering of a reactive surface by graphene may result in the weakening of the oxidation protection. Similar processes have also been confirmed at the edges of relatively larger holes. This serious limitation caused by defects can be avoided by multilayer coatings.

\section{CONCLUSIONS}

In conclusion, we have demonstrated that a continuous coating of pristine graphene on reactive surfaces can provide excellent protection from oxidation of reactive surfaces at the nanoscale. Even if the binding of oxygen atom at low coordinated carbon atoms at the vacancy is rather strong, the barrier for their penetration to the reactive surface under graphene is low. Therefore discontinuities in graphene coating or defects, such as vacancies or holes, weaken the protection from oxidation by creating spots of a low oxidation barrier. This limitation can be circumvented by coating with bilayer graphene or, preferably, graphene sheets comprising a few graphene layers, which provide even more effective protection. At the macroscale, our results suggest that graphene additives can improve the strength of antioxidant paints. A graphene coating which is thin, at the atomic scale, can also serve as a natural barrier between the environment and solid surfaces of other elements.

\section{ACKNOWLEDGMENTS}

This work was supported by TUBITAK through Grant No. 108T234. All computational resources were provided by TUBITAK ULAKBIM, High Performance and Grid Computing Center (TR-Grid e-Infrastructure). The authors acknowledge useful discussions with Prof. J. Schrier. S.C. acknowledges the partial support of TUBA, the Academy of Science of Turkey. *ciraci@fen.bilkent.edu.tr

${ }^{1}$ F. J. Derbyshire, A. E. B. Presland, and D. L. Trimm, Carbon 13, 111 (1975).

${ }^{2}$ M. Eizenberg and J. M. Blakely, Surface Sci. 82, 228 (1979).

${ }^{3}$ J. C. Hamilton and J. M. Blakely, Surface Sci. 91, 199 (1980).

${ }^{4}$ J. W. May, Surface Sci. 17, 267 (1969).

${ }^{5}$ K. S. Novoselov, A. K. Geim, S. V. Morozov, D. Jiang, Y. Zhang, S. V. Dubonos, I. V. Grigorieva, and A. A. Firsov, Science 306, 666 (2004).

${ }^{6} \mathrm{~J}$. Wintterlin and M.-L. Bocquet, Surface Sci. 603, 1841 (2009).

${ }^{7}$ C. Mattevi, H. Kim, and M. Chhowalla, J. Mater. Chem. 21, 3324 (2011).

${ }^{8}$ Y. S. Dedkov, M. Fonin, U. Rudiger, and C. Laubschat, Appl. Phys. Lett. 93, 022509 (2008); Y. S. Dedkov, M. Fonin, and C. Laubschat, ibid. 92, 052506 (2008).

${ }^{9}$ B. Borca, F. Calleja, J. J. Hinarejos, A. L. Vazquez de Parga, and R. Miranda, J. Phys. Condens. Matter 21, 134002 (2009).

${ }^{10}$ S. Gadipelli, I. Calizo, J. Ford, G. Cheng, A. R. H. Walker, and T. Yildirim, J. Mater, Chem. 21, 16057 (2011).

${ }^{11}$ S. Chen, L. Brown, M. Levendorf, W. Cai, S.-Y. Ju, J. Edgeworth, X. Li, C. W. Magnuson, A. Velamakanni, R. D. Piner, J. Kang, J. Park, and R. S. Ruoff, ACS Nano 5, 1321 (2011).

${ }^{12}$ J. Cho, L. Gao, J. Tian, H. Cao, W. Wu, Q. Yu, E. N. Yitamben, B. Fisher, J. R. Guest, Y. P. Chen, and N. P. Guisinger, ACS Nano 5, 3607 (2011).
${ }^{13}$ N. A. Vinogradov, K. Schulte, M. L. Ng, A. Mikkelsen, E. Lundgren, N. Mårtensson, and A. B. Preobrajenski, J. Phys. Chem. C 115, 9568 (2011).

${ }^{14}$ G. Kresse and J. Hafner, Phys. Rev. B 47, 558 (1993).

${ }^{15}$ G. Kresse and J. Furthmüller, Phys. Rev. B 54, 11169 (1996).

${ }^{16}$ J. P. Perdew, K. Burke, and M. Ernzerhof, Phys. Rev. Lett. 77, 3865 (1996).

${ }^{17}$ S. Grimme, J. Comput. Chem. 27, 1787 (2006).

${ }^{18}$ P. E. Blochl, Phys. Rev. B 50, 17953 (1994).

${ }^{19}$ J. K. Grepstad, P. O. Gartland, and B. J. Slagsvold, Surface Sci. 57, 348 (1976).

${ }^{20}$ G. Henkelman, A. Arnaldsson, and H. Jónsson, Comput. Mater. Sci. 36, 254 (2006).

${ }^{21}$ Z. Wei, D. Wang, S. Kim, S.-Y. Kim, Y. Hu, M. K. Yakes, A. R. Laracuente, Z. Dai, S. R. Marder, C. Berger, W. P. King, W. A. de Heer, P. E. Sheehan, and E. Riedo, Science 328, 1373 (2010).

${ }^{22}$ O. O. Ekiz, M. Urel, H. Guner, A. K. Mizrak, and A. Dana, ACS Nano 5, 2475 (2011).

${ }^{23}$ L. Signorini, L. Pasquini, L. Savini, R. Carboni, F. Boscherini, E. Bonetti, A. Giglia, M. Pedio, N. Mahne, and S. Nannarone, Phys. Rev. B 68, 195423 (2003).

${ }^{24}$ I. P. Batra and S. Ciraci, Phys. Rev. B 29, 6419 (1984).

${ }^{25}$ A. Kiejna, Phys. Rev. B 68, 235405 (2003).

${ }^{26}$ P. Giannozzi, S. Baroni, N. Bonini et al., J. Phys.: Condens. Matter 21, 395502 (2009).

${ }^{27}$ M. Vanin, J. J. Mortensen, A. K. Kelkkanen, J. M. Garcia-Lastra, K. S. Thygesen, and K. W. Jacobsen, Phys. Rev. B 81, 081408 (2010). 
${ }^{28}$ A. Buldum, D. M. Leitner, and S. Ciraci, Phys. Rev. B 59, 16042 (1999).

${ }^{29}$ H. Sevincli, S. Mukhopadhyay, R. T. Senger, and S. Ciraci, Phys. Rev. B 76, 205430 (2007).

${ }^{30}$ M. Topsakal, E. Akturk, H. Sevincli, and S. Ciraci, Phys. Rev. B 78, 235435 (2008).
${ }^{31}$ A. Hashimoto, K. Suenaga, A. Gloter, K. Urlta, and S. Iijima, Nature (London) 430, 870 (2004).

${ }^{32}$ S. M. Lee, Y. H. Lee, Y. G. Hwang, J. R. Hahn, and H. Kang, Phys. Rev. Lett. 82, 217 (1999).

${ }^{33}$ J. M. Carlsson, F. Hanke, S. Linic, and M. Scheffler, Phys. Rev. Lett. 102, 166104 (2009). 